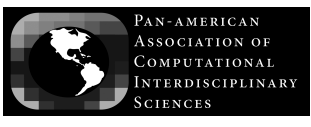

Journal of Computational Interdisciplinary Sciences (2009) 1(2): 165-173

(C) 2009 Pan-American Association of Computational Interdisciplinary Sciences

ISSN 1983-8409

http://epacis.org

\title{
A new numerical code for the Bondi problem
}

\author{
Henrique P. de Oliveira and E.L. Rodrigues
}

Manuscript received on October 20, 2008 / accepted on January 10, 2009

\begin{abstract}
We present the first numerical code based on the Galerkin method to integrate the field equations of the Bondi problem. The Galerkin method is a spectral method whose main feature is to provide high accuracy with moderate computational effort. Several numerical tests were performed to verify the issues of convergence, stability and accuracy with promising results. This code opens up several possibilities of applications in more general scenarios for studying the evolution of spacetimes with gravitational waves.
\end{abstract}

Keywords: Numerical Relativity, Galerkin method, gravitational radiation. 


\section{INTRODUCTION}

The General Theory of Relativity [1] is the modern theory for the gravitational field. According to General Relativity, gravity is understood as the curvature of the spacetime continuum due to the presence of matter fields such as fluids, electromagnetic field, scalar field, etc. The so called Einstein field equations govern the behavior of gravitational systems and are written in the following way

$$
R_{\alpha \beta}-\frac{1}{2} g_{\alpha \beta} R=\frac{8 \pi G}{c^{4}} T_{\alpha \beta},
$$

where the Greek indices vary from 0 to 3 indicating temporal, $x^{0}$ and spatial coordinates, $\left(x^{1}, x^{2}, x^{3}\right) . \quad R_{\alpha \beta}$ and $R=$ $g_{\alpha \beta} R^{\alpha \beta}$ are the Ricci tensor and the scalar of curvature, respectively; $g_{\alpha \beta}\left(x^{\mu}\right)$ is the metric tensor, $G$ is the gravitational constant, $c$ is the vacuum speed of light and $T_{\alpha \beta}$ is the energymomentum tensor of the matter field. The left hand side accounts for the geometry of the spacetime through expressions formed by the metric tensor, their first, $\partial g_{\alpha \beta} / \partial x^{\mu} \equiv g_{\alpha \beta, \mu}$, and second derivatives $\partial^{2} g_{\alpha \beta} / \partial x^{\mu} \partial x^{\nu} \equiv g_{\alpha \beta, \mu \nu}$, whereas the right hand side describes the matter content that curves the spacetime continuum producing, in this way the gravitational field. In the Newtonian limit, that is for weak gravitational fields and low velocities, the Einstein equations reduce to $\nabla^{2} \Phi=4 \pi G \rho$, where $\Phi$ is the gravitational potential.

The most significant feature of the field equations is that it constitutes a set of ten nonlinear coupled partial differential equations for the metric tensor, possibly the most difficult set of differential equations of mathematical physics. As a consequence, few exact and approximate analytical solutions of the field equations are known, in general obtained after introducing highly simplified assumptions, and therefore not suitable to describe systems of physical or astrophysical relevance that demand much less simplified description of their gravitational and matter fields. In such systems the features provided by the nonlinearities of field equations play the relevant role producing, for instance, chaotic and turbulent behavior, in another words, a complex behavior. In this sense, we can affirm that complex systems are ubiquitous in General Relativity.

One of the most important problems of General Relativity is the emission of gravitational waves by a realistic bounded source, for instance as the result of collapse and formation of a neutron star or even a black hole. In their seminal works Bondi et al. [2, 3] have launched the basis for a detailed description for the dynamics of the exterior axisymmetric spacetime of a bounded source undergoing a process of gravitational wave emission.
They have established the suitable form of the metric, the field equations and also exhibited the set of permissable coordinate transformations that preserve the nature of the metric of what is known by the Bondi problem. Their most important result was the introduction of the news function that determines the rate of mass Ioss due to gravitational wave extraction, along with providing an invariant characterization of the presence of gravitational radiation. Nonetheless, another important pioneering aspect of this work was the presentation of the field equations in the scheme of characteristics [4] more than ten years before the formalization of the Cauchy approach [5] of Einstein equations.

Due to the complicated form of the Bondi equations there are no analytical solutions describing the evolution of a nonstationary axisymmetric spacetime endowed with gravitational waves. Therefore, the main features of the dynamics of the Bondi problem can only be determined through numerical simulations. The first numerical code applied to integrate the Bondi equations was developed by Issacson, Welling and Winicour [6], and later improved by a version that avoided instabilities near the vertex [7]. Other groups have also presented distinct strategies for constructing their codes as, for instance, using the tetrad formalism [8] or combining Cauchy and characteristics evolution that allowed the extension of the Bondi problem to full axisymmetry [9]. A detailed description of the numerical schemes used for the characteristic evolution can be found in Ref. [4], but the common feature shared by all of these codes is that they were constructed using finite difference techniques.

Spectral methods $[10,11]$ represent an alternative strategy to solve numerically partial differential equations (PDEs), and are based on the idea of approximating the solution as a finite series of a convenient set of analytical basis functions. As a consequence, any spectral method transforms a PDE or a system of PDEs into a finite system of ordinary differential equations for the coefficients that multiply the basis functions, where it is expected that the greater is the number of coefficients, or the number of ordinary differential equations, more accurate is the approximate solution. There are two main attractive features of the spectral methods:

(i) a considerable economy of the computational resources to achieve a given accuracy if compared with the finite difference techniques;

(ii) the possibility of selecting a coordinate system adapted to the geometry of the problem under consideration allowing an exact treatment of pseudo-singularities present in the chosen coordinates. 
As a result the use of spectral methods has been increased considerably in the last years and figures as viable strategy for $\mathrm{Nu}-$ merical Relativity [12]. In particular, amongst the several types of spectral methods we shall focus in the Galerkin method [13], whose main feature is that each basis function is selected so as to satisfy automatically the boundary conditions.

In this work we have developed the first numerical code based on the Galerkin method $[10,11,13]$ to integrate the field equations of the Bondi problem. In Section 2 the field equations as well as a brief description of their relevant aspect are presented. The Galerkin method is applied to the Bondi problem (Section 3) whose the most important step is the choice of convenient basis functions for the metric functions. In Section 4 we have presented some code tests to check the accuracy of the our code. Finally, in Section 5 we discuss some perspectives of the present work.

\section{THE BONDI PROBLEM}

The metric for axisymmetric and asymptotically flat spacetimes corresponding to the Bondi problem [3] takes the form

$$
\begin{gathered}
d s^{2}=\left(\frac{V}{r} \mathrm{e}^{2 \beta}-U^{2} r^{2} \mathrm{e}^{2 \gamma}\right) d u^{2}+2 \mathrm{e}^{2 \beta} d u d r \\
+2 U r^{2} \mathrm{e}^{2 \gamma} d u d \theta-r^{2}\left(\mathrm{e}^{2 \gamma} d \theta^{2}+\mathrm{e}^{-2 \gamma} \sin ^{2} \theta d \phi^{2}\right),
\end{gathered}
$$

where $u$ is the retarded time coordinate for which the outgoing null cones are denoted by $u=$ constant. The radial coordinate $r$ is chosen so that the surfaces of constant $(u, r)$ have area $4 \pi r^{2}$; the angular coordinates $(\theta, \phi)$ are constant along the outgoing null geodesics. The functions $\gamma, \beta, U$ and $V$ depend on the coordinates $u, r, \theta$ and satisfy the vacuum field equations $R_{\mu \nu}=0$ organized in three hypersurface equations and one evolution equation, respectively, written as

$$
\begin{gathered}
\beta_{, r}=\frac{1}{2} r\left(\gamma_{, r}\right)^{2} \\
{\left[r^{4} \mathrm{e}^{2(\gamma-\beta)} U_{, r}\right]_{, r}=2 r^{2}\left[r^{2}\left(\frac{\beta}{r^{2}}\right)_{, r \theta}-\frac{\left(\sin ^{2} \theta \gamma\right)_{, r \theta}}{\sin ^{2} \theta}+2 \gamma_{, r} \gamma_{, \theta}\right]} \\
V_{, r}=-\frac{1}{4} r^{4} \mathrm{e}^{2(\gamma-\beta)}\left(U_{, r}\right)^{2}+\frac{\left(r^{4} \sin \theta U\right)_{, r \theta}}{2 r^{2} \sin \theta}+\mathrm{e}^{2(\beta-\gamma)}\left[1-\frac{\left(\sin \theta \beta_{, \theta}\right)_{, \theta}}{\sin \theta}+\gamma_{, \theta \theta}\right. \\
\left.+3 \cot \theta \gamma_{, \theta}(\beta, \theta)^{2}-2 \gamma_{, \theta}\left(\gamma_{, \theta}-\beta, \theta\right)\right], \\
4 r(r \gamma)_{, u r}=\left\{2 r \gamma_{, r} V-r^{2}\left[2 \gamma_{, \theta} U+\sin \theta\left(\frac{U}{\sin \theta}\right)_{, \theta}\right]\right\}_{, r}-2 r^{2} \frac{\left(\gamma_{, r} U \sin \theta\right)_{, \theta}}{\sin \theta}+\frac{1}{2} r^{4} \mathrm{e}^{2(\gamma-\beta)}\left(U_{, r}\right)^{2} \\
+2 \mathrm{e}^{2(\beta-\gamma)}\left[\left(\beta_{, \theta}\right)^{2}+\sin \theta\left(\frac{\beta, \theta}{\sin \theta}\right)_{, \theta}\right] .
\end{gathered}
$$

The subscripts $r, u$ and $\theta$ denote partial derivatives with respect to these coordinates. Notice that the evolution equation is the only one that has a derivative with respect to $u$, while the hypersurface equations contain only derivatives in the null hypersurfaces $u=$ constant. In order to guarantee regularity of the spacetime at the origin $r=0$ we have

$$
\gamma \sim \mathcal{O}\left(r^{2}\right), \quad \beta \sim \mathcal{O}\left(r^{4}\right), \quad U \sim \mathcal{O}(r), \quad V \sim r+\mathcal{O}\left(r^{3}\right) . \quad(7)
$$

Also, by imposing smoothness of the axis it is necessary that

$$
\frac{\gamma}{\sin ^{2} \theta}, \quad \frac{U}{\sin \theta}
$$

be continuous at $\theta=0, \pi$.

As pointed out by Bondi et al. [3] the above set of equations has a nice hierarchical structure typical of the characteristic formulation of the Einstein's equations [4]. Once the initial data is given, in other words when the function $\gamma$ is specified on the initial null surface $u=u_{0}$, the constraint equations (3), (4) and (5) determine the metric functions $\beta, U$ and $V$ (modulo integration constants) on the null surface $u=u_{0}$. From these results, the evolution equation (6) provides $\gamma_{, u}$ on $u=u_{0}$, and consequently allows the determination of $\gamma$ on the next null surface $u=u_{1}$, and the whole cycle repeats providing the evolution of the spacetime.

\section{THE GALERKIN APPROACH}

An alternative strategy adopted in finite difference technique of representing a function by numbers in a grid, the Galerkin 
method [13] or any other spectral method [10] assume that the function under consideration can be well approximated by a series of a suitable set of basis formed by known analytical functions, as for instance, Legendre or Chebyshev polynomials. According to the Galerkin method the each basis functions must satisfy the boundary conditions. Thus, let us construct a convenient Galerkin decomposition for the metric function $\gamma$ using, as our guides, the boundary condition at the origin given by Eq. (7), and the regularity of $\gamma / \sin ^{2} \theta$ at $\theta=0, \pi$. We have come up with the following decomposition

$\gamma(u, r, x)=\left(1-x^{2}\right) \sum_{j=0}^{N_{x}} \sum_{k=0}^{N_{r}} a_{k j}(u) \Psi_{k}^{(\gamma)}(r) P_{j}(x)$,

where for the sake of convenience we have introduced $x=$ $\cos \theta . N_{r}$ and $N_{x}$ are the truncation orders of the radial and angular expansions, respectively; $a_{k j}(u), k=0,1, \ldots, N_{r}$, $j=0,1, \ldots, N_{x}$ are the modal coefficients that depend on the retarded time $u$, and the Legendre polynomials $P_{j}(x)$ were selected as the angular basis functions due to the regularity of

$$
\frac{\gamma}{\sin ^{2} \theta}=\frac{\gamma}{\left(1-x^{2}\right)} \text { at } x= \pm 1 \text {. }
$$

Concerning to the radial expansion, $\Psi_{k}^{(\gamma)}(r)$ is the basis function of order $k$ constructed using a suitable linear combination of the rational Chebyshev polynomials [11] (see Appendix) in order to assure the fulfilment of the boundary condition at the origin, that is $\Psi_{k}^{(\gamma)}(r) \sim \mathcal{O}\left(r^{2}\right)$ near $r=0$ for all $k$. In addition the choice of $\Psi_{k}^{(\gamma)}(r)$ provides the correct asymptotic expression for $\gamma$ at future null infinity by expanding Eq. (9) in inverse powers of the radial coordinate, or

$$
\gamma=K(u, x)+\frac{c(u, x)}{r}+\mathcal{O}\left(r^{-2}\right),
$$

where the functions $K(u, x), c(u, x)$ are expressed in terms of the modal coefficients $a_{k j}(u)$ and the Legendre polynomials. It is important to emphasize that a global representation of the metric function $\gamma(u, r, x)$ as given by Eq. (9), and the other metric functions (see below) allow us to access directly important quantities defined at future null infinity that determines the amount of mass and momentum radiated away by gravitational waves.

Now, the next step is to provide appropriate decompositions for the metric functions $\beta, U$ and $V$. Before establishing these decompositions, we follow Ref. [14] and introduce the function $S(u, r, x)$ related to $V$ by $V \equiv r+r^{2} S$. In this case, the behavior of $S$ near the origin is the same of $U$ and therefore the radial basis for both decompositions will be the same. The Galerkin decompositions for the metric functions $\beta, U$ and $S$ are

$$
\begin{gathered}
\beta(u, r, x)= \\
\left(1-x^{2}\right)^{2} \sum_{j=0}^{N_{x}^{\beta}} \sum_{k=0}^{N_{r}^{\beta}} c_{k j}(u) \Psi_{k}^{(\beta)}(r) P_{j}(x) \\
U(u, r, x)=\sqrt{1-x^{2}} \sum_{j=0}^{N_{x}^{U}} \sum_{k=0}^{N_{r}^{U}} b_{k j}(u) \Psi_{k}^{(U)}(r) P_{j}(x) \\
S(u, r, x)=\sum_{j=0}^{N_{x}^{S}} \sum_{k=0}^{N_{r}^{S}} s_{k j}(u) \Psi_{k}^{(U)}(r) P_{j}(x) .
\end{gathered}
$$

Notice that the angular basis functions are the Legendre polynomials, and each radial basis functions (cf. Appendix) satisfies the boundary conditions given by Eqs. (7) and (8) and also provide the correct asymptotic forms for the metric. The truncation orders for each Galerkin decomposition are indicated by $\left(N_{r}^{\beta}, N_{x}^{\beta}\right)$, $\left(N_{r}^{U}, N_{x}^{U}\right)$ and $\left(N_{r}^{S}, N_{x}^{S}\right)$. The corresponding asymptotic expressions for the above metric functions are

$$
\begin{aligned}
\beta= & H(u, x)+\mathcal{O}\left(r^{-2}\right) \\
U= & L(u, x)+\mathcal{O}\left(r^{-1}\right) \\
S= & S_{0}(u, x)+\frac{S_{-1}(u, x)}{r} \\
& -\frac{2 \mathrm{e}^{2 H} M(u, x)}{r^{2}}+\mathcal{O}\left(r^{-3}\right),
\end{aligned}
$$

where it can be shown that the $S_{0}=-\partial\left(L \sqrt{1-x^{2}}\right) / \partial x$, $S_{-1}$ is related to the functions $H, K, L$ [6], and $M(u, x)$ is known as the Bondi mass aspect [3]. As we are going to see these asymptotic expressions will be of fundamental importance for the evaluation of the Bondi mass and the news function. In the standard Bondi coordinates $K=L=H=0$ [3].

The next step towards the construction of our algorithm to solve numerically the Bondi equations is to substitute the decompositions of the metric functions into the field equations. We begin by inserting Eqs. (9) and (11) into the hypersurface Eq. (3) yielding the following residual equation

$$
\begin{gathered}
\operatorname{Res}_{\beta}(u, r, x)=\sum_{j=0}^{N_{x}^{\beta}} \sum_{k=0}^{N_{r}^{\beta}} c_{k j}(u) \Psi_{k, r}^{(\beta)} P_{j}(x) \\
-\frac{1}{2} r\left(\sum_{j=0}^{N_{x}} \sum_{k=0}^{N_{r}} a_{k j}(u) \Psi_{k, r}^{(\gamma)} P_{j}(x)\right)^{2} .
\end{gathered}
$$


Since the decompositions for $\beta$ and $\gamma$ are indeed approximations, the residual equation does not vanish exactly. Nevertheless, it is expected that the residual approaches to zero as the truncation orders are increased, or $\operatorname{Res}_{\beta} \rightarrow 0$ as $N r, N_{x}, N_{x}^{\beta}$, $N_{r}^{\beta} \rightarrow \infty$. According to the Galerkin method the scalar products, or the projections of the residual equation onto all radial and angular basis functions must vanish. These operations are schematically indicated by

$$
\begin{gathered}
\left\langle\operatorname{Res}_{\beta}, \Psi_{k}^{(\beta)} P_{j}(x)\right\rangle= \\
\int_{-1}^{1}\left(\int_{0}^{\infty} \operatorname{Res}_{\beta}(u, r, x) \Psi_{k}^{(\beta)} P_{j}(x) \frac{d r}{\sqrt{r}(r+1)}\right) d x=0,
\end{gathered}
$$

where $k=0,1, \ldots, N_{r}^{\beta}, j=0,1, \ldots, N_{x}^{\beta}$, and the denominator is the weight function of the internal product of Chebyshev polynomials. After the integrations we obtain a set of $\left(N_{x}^{\beta}+1\right) \times\left(N_{r}^{\beta}+1\right)$ algebraic equations connecting the modal coefficients $c_{k j}$ with $a_{k j}$. Therefore, the hypersurface Eq. (3) is transformed into an algebraic system of equations.

Repeating the same procedure taking into account the hypersurface equations (4) and (5) for the functions $U$ and $S$, respectively, we ended up with two set of algebraic equations: the first set connects the modal coefficients $b_{k j}$ with the modal coefficients $a_{k j}$ and $c_{k j}$, and the second set relates the modal coefficients $s_{k j}$ with $a_{k j}, b_{k j}$ and $c_{k j}$. Finally, after imposing that the projections of the residual equation corresponding to the evolution equation into all $\Psi_{k}^{(\gamma)}$ and $P_{j}(x)$, a system of $\left(N_{x}+1\right) \times\left(N_{r}+1\right)$ nonlinear ordinary differential equations for the modal coefficients $a_{k j}$ is obtained. This last set of equations is schematically represented as

$$
\dot{a}_{k j}(u)=F_{k j}\left(a_{l m}, b_{l m}, c_{l m}, s_{l m}\right),
$$

where dot means derivative with respect to $u$ and $k=$ $0,1, \ldots, N_{r}, j=0,1, \ldots, N_{x}$. Therefore the Bondi problem is reduced to a system of ordinary differential equations, or a dynamical system, along with three sets of algebraic equations. In order to integrate the Bondi equations, or equivalently, the dynamical system (19), the initial conditions $a_{l m}\left(u_{0}\right), b_{l m}\left(u_{0}\right)$, $c_{l m}\left(u_{0}\right), s_{l m}\left(u_{0}\right)$ must be given. To accomplish with such a task we have first to determine the Galerkin decomposition of the initial data $\gamma_{0}(r, x)=\gamma\left(u_{0}, r, x\right)$ that will fix the set of $a_{l m}\left(u_{0}\right)$. The initial values of the modal coefficients $b_{l m}\left(u_{0}\right)$ are determined using the first set of algebraic equations obtained from the projections indicated by (18); considering these results, the second and third sets of algebraic equations determine all $c_{l m}\left(u_{0}\right)$ and $s_{l m}\left(u_{0}\right)$, respectively. In this way, using these initial modal coefficients the dynamical system (19) determines the modal coefficients $a_{l m}$ in the next step, or in the next hypersurface $u+\delta u$, and the whole process repeats, providing the numerical solution of the system.

\section{CODE TESTS}

We now proceed to present the code tests $[7,14,15]$ to check the issues of stability, accuracy and convergence of our algorithm. In the first test a small amplitude gravitational wave is evolved and compared it with the corresponding exact solution known from the linearized field equations. The other test, and probably most important, is to check the global energy conservation given by the Bondi formula that connects the loss of mass with the total energy emitted by gravitational waves, where this last quantity is evaluated using the news function.

In order to carry out these tests we have derived a simplified version of the dynamical system (19), where the nonlinear effects are restricted to be of second order in the following way. We have chosen the metric function $\gamma$ small in the sense of $\left|\gamma_{\max }\right| \lesssim$ $10^{-3}$, and with associated modal coefficients $\left|a_{k j}\right| \lesssim 10^{-3}$. From the hypersurface equations it follows that

$$
\mathcal{O}(\beta) \sim \mathcal{O}\left(\gamma^{2}\right), \mathcal{O}(U) \sim \mathcal{O}(S) \sim \mathcal{O}(\gamma),
$$

and consequently similar relations expressed in terms of the modal coefficients $a_{k j}, b_{k j}, c_{k j}$ and $s_{k j}$ arise. Then, we have generated a dynamical system in which the terms smaller or of order, say $\mathcal{O}\left(\beta^{2}\right), \mathcal{O}\left(\gamma^{3}\right)$, were neglected. The main motivation for this procedure is to provide a fast and simple algorithm whose accuracy and convergence can be tested without much computational effort, and at the same time catching the main features of nonlinear effects.

In the linear regime $\beta \simeq 0$ and $V \simeq r$, and the weak disturbances of the spacetime are described by the functions $\gamma$ and $U$. It can be shown $[3,7]$ that the linearized equations are equivalent to a flat scalar wave equation $\square \Phi=0$, where $\Phi(u, r, x)$ is related to $\gamma$ and $U$ [7], and hence from the exact solution of the wave equation the corresponding exact expressions for $\gamma$ and $U$ are determined. Following this procedure the quadrupole solution denoted by $\gamma_{[2]}$ is expressed as

$$
\gamma_{[2]}(u, r, x)=\frac{6 A_{0}\left(1-x^{2}\right) r^{2}(u+r+1)}{(u+1)^{4}(u+2 r+1)^{3}},
$$

where $A_{0}$ is the free amplitude of the linearized equations.

Now we chose a very small value for the amplitude, say $A_{0}=5.0 \times 10^{-4}$, in order that the nonlinear code be able 
to reproduce the linear solution. The initial values of the modal coefficients $a_{k j}(u=0)$ are determined from the above initial data after establishing the following residual equation

$$
\begin{gathered}
\text { Residual }= \\
\bar{\gamma}_{[2]}(0, r, x)-\sum_{k, j=0} a_{k j}(0) \Psi_{k}^{(\gamma)}(r) P_{j}(x),
\end{gathered}
$$

where $\gamma=\left(1-x^{2}\right) \bar{\gamma}$, and impose that their projections into the radial and angular basis functions vanishes, or $\left\langle\right.$ Residual, $\left.\Psi_{m}^{(\beta)} P_{n}(x)\right\rangle=0$ for $m=0,1, \ldots, N_{r}$ and $n=0,1, \ldots, N_{x}$. In this way, a set of $\left(N_{r}+1\right) \times\left(N_{x}+1\right)$ equations is generated that can be solved for the initial values $a_{k j}(0)$.

In Figure 1 we illustrate the excellent agreement between the the exact (continuous line) and the approximate (boxes) solutions evaluated at several times for $N_{r}=6$ and $N_{x}=4$, where for the sake of convenience we have introduced the variable $y$ related to the radial coordinate through $r=y /(1-y)$ such that $0 \leq y \leq 1$ corresponds to the $0 \leq r<\infty$. The decay of the gravitational wave shear $\bar{\gamma}_{[2]}$ evaluated at future null infinity $(y=1)$, or the function

$$
\bar{K}(u, x)=\frac{K}{\left(1-x^{2}\right)}
$$

(cf. Eq. (10)) is shown in Figure 2(a), where for the quadrupole mode $\bar{K}=\bar{K}(u)$. It can be seen from Figure 2(b) the evolution of $\left|\bar{K}_{\text {exact }}-\bar{K}_{\text {numer }}\right|$ with increasing truncation orders $\left(N_{r}, N_{x}\right)$. As expected the greater is the truncation orders the numerical solution becomes more close to the exact one.

At this point it is useful to comment the role played by the choice of the truncation orders associated to the Galerkin decompositions of the metric functions. The truncation orders $N_{r}, N_{x}$ of the radial and angular expansions of $\gamma$, respectively, are our seeds for which the corresponding truncation orders $N_{r}^{U}, N_{x}^{U}$, $N_{r}^{S}, \ldots$ will be related with through the constrained equations (3)-(5). We have found that the stability of the code, as far as the evolution of weak gravitational waves are concerned, is achieved if the following relations between the truncation orders are satisfied, $N_{x}^{U} \geq N_{x}+1$ and $N_{x}^{S} \geq N_{x}+2$ for the angular part, and $N_{r}^{U} \geq N_{r}, N_{r}^{S} \geq N_{r}$ associated to the radial decomposition. For the sake of convenience we have considered in all numerical experiments the following radial and truncation orders: $N_{r}^{U}=N_{r}+2, N_{r}^{S}=N_{r}+1, N_{r}^{\beta}=N_{r}-1$, and $N_{x}^{U}=N_{x}+1, N_{x}^{S}=N_{x}+2, N_{x}^{\beta}=N_{x}-1$.

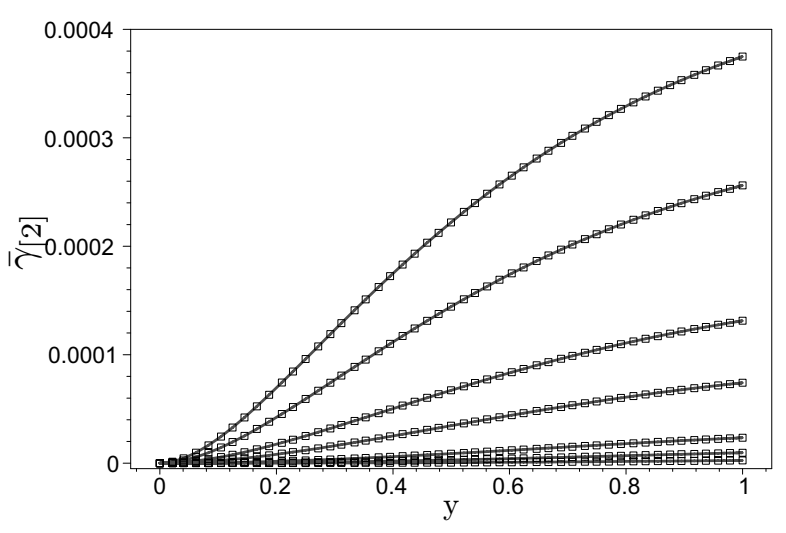

Figure 1 - Illustration of the agreement between the exact (continuous line) and approximate (boxes) expressions for the quadrupole mode (20). The curves from the top to bottom are evaluated at $u=0,0.1,0.3,0.5,1.0,1.5$ and 2.5 , respectively. The truncation orders are $N_{r}=6, N_{x}=4$ and $N_{r}^{U}=7, N_{x}^{U}=5$ for the Galerkin decompositions of $\gamma$ and $U$, respectively.

The last and probably most important test is to check the global energy conservation derived from the Bondi formula. Following Gomez et al. [7] the expression for global energy conservation in the gauge under consideration is given by

$$
\begin{aligned}
C(u)= & M_{B}(u)-M_{B}\left(u_{0}\right) \\
& +\frac{1}{2} \int_{u_{0}}^{u} d u \int_{-1}^{1} \frac{\mathrm{e}^{2 H}}{\omega} N^{2} d x=0,
\end{aligned}
$$

that is obtained after integrating the Bondi formula. Here $M_{B}(u)$ is the Bondi mass, $H(u, x)$ is given by Eq. (14) and $N$ is the news function. The conformal $\omega$ depends on the gauge we are adopting and arises by connecting the metric of the two geometry of a unit sphere in the standard Bondi coordinates with the similar expression in our coordinate system, or

$$
\begin{aligned}
d \hat{s}_{B}^{2} & =d \theta_{B}^{2}+\sin ^{2} \theta_{B} d \phi_{B}^{2} \\
& =\omega^{2}\left(\mathrm{e}^{2 K} d \theta^{2}+\sin ^{2} \theta \mathrm{e}^{-2 K} d \phi^{2}\right)
\end{aligned}
$$

yielding

$$
\omega=\frac{2 \mathrm{e}^{K}}{(1+x) \mathrm{e}^{\Delta}+(1-x) \mathrm{e}^{-\Delta}},
$$

where

$$
\Delta=\int_{0}^{x} \frac{\mathrm{e}^{2 K}-1}{1-x^{2}} d x .
$$

The news function $N(u, r, x)$ can be written as [16]

$$
\begin{aligned}
N= & \mathrm{e}^{-2 H} c_{, u}-\mathrm{e}^{-2 H} \frac{\left(\sqrt{1-x^{2}} c^{2} L\right)^{\prime}}{2 c} \\
& +\frac{1}{2} \mathrm{e}^{-2(K+H)} \omega\left(1-x^{2}\right)\left[\frac{\left(\omega \mathrm{e}^{2 H}\right)^{\prime}}{\omega^{2}}\right]^{\prime}
\end{aligned}
$$



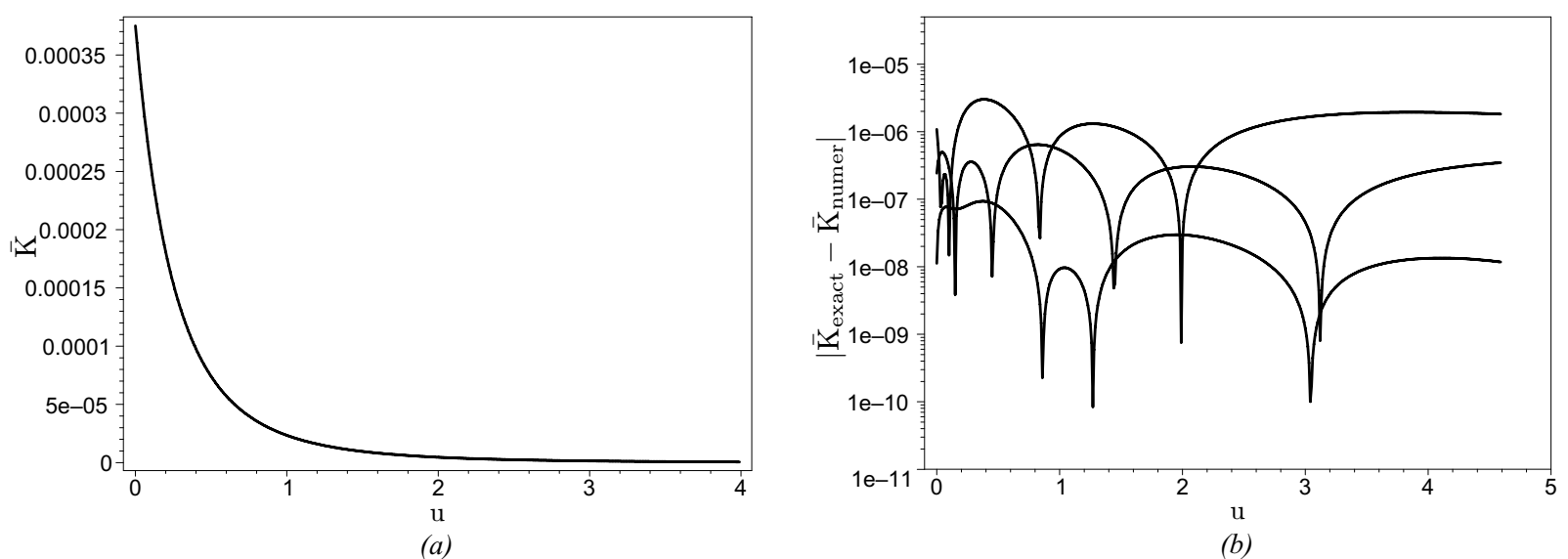

(b)

Figure 2 - (a) Decay of the exact shear $\bar{K}(u, x)$. (b) Log-linear plot of the absolute error $\left|\bar{K}_{\text {exact }}-\bar{K}_{\text {numer }}\right|$ with the curves from top to below correspond to the truncation orders $N_{r}=3, N_{x}=3, N_{r}=4, N_{x}=4$ and $N_{r}=6, N_{x}=4$, respectively.

The Bondi mass depends directly on the mass aspect $M$ (cf. Eq. (16)) and the terms arising from the gauge under consideration. According to Ref. [16] the Bondi mass $M_{B}(u)$ is given by

$$
\begin{gathered}
M_{B}(u)=\int_{-1}^{1} \omega^{-1}\left\{\frac{1}{2} M+\frac{1}{4} \mathrm{e}^{-2 K}\left[\left(1-x^{2}\right) c^{\prime \prime}\right.\right. \\
\left.-4 x c^{\prime}-2 c\right]-\mathrm{e}^{-2 K} c^{\prime}\left(H^{\prime}+K^{\prime}\right)\left(1-x^{2}\right) \\
-\mathrm{e}^{-2 K} c\left(H^{\prime 2}-2 H^{\prime} K^{\prime}-K^{\prime 2}\right)\left(1-x^{2}\right) \\
-\frac{1}{2} \mathrm{e}^{-2 K} c\left[\left(1-x^{2}\right)\left(H^{\prime \prime}-K^{\prime \prime}\right)\right. \\
\left.\left.-4 x\left(H^{\prime}+K^{\prime}\right)\right]\right\} d x .
\end{gathered}
$$

The standard Bondi frame [3] is characterized by a choice of a coordinate system for which $H=K=L=0$ and consequently $\omega=1$. Also, in this frame the Bondi mass depends only on $M$ and Eq. (22) agrees with the original expression for the Bondi formula [3]. As an important remark concerning the Galerkin treatment of the field equations is that the functions $H, K, L$ and $M$ can be read off directly from the asymptotic expressions for the metric functions as presented by Eqs. (10), (14)-(16).

Before verifying the global energy conservation (22), we first evaluate the initial Bondi mass $M_{B}(0)$ corresponding to the following initial data

$$
\begin{gathered}
\gamma_{0}(r, x)= \\
\gamma_{[2]}(0, r, x)\left[1+\frac{6\left(1-x^{2}\right) r^{2}(u+r+1)}{(u+1)^{4}(u+2 r+1)^{3}}\right],
\end{gathered}
$$

where $\gamma_{[2]}(0, r, x)$ is the quadrupole mode given by Eq. (4). This initial data can be understood as the quadrupole mode plus a self-interaction term (note that the term inside the parenthesis is the quadrupole mode with $A_{0}=1$ ). We have set $A_{0}=0.025$ considering several truncation orders and the Bondi mass evaluated for distinct truncation orders are shown in Figure 3(a). Notice that the sequence of $M_{B}\left(u_{0}=0\right)$ signalizes a clear the convergence towards the actual value for the initial Bondi mass. The next step consisted in showing the evolution of the relative error in energy conservation, $|C(u)| / M_{B}(0) \times 100$, with the truncation orders $N_{r}=5, N_{x}=4$ and $N_{r}=7, N_{x}=5$. As indicated in Figure $3(\mathrm{~b})$, the energy conservation is attained to $1.3 \%$ and $0.45 \%$ accuracies, respectively.

\section{DISCUSSION}

In this paper we have constructed an alternative code for the evolution of the Bondi problem based on the Galerkin method. The important feature of a satisfactory accuracy with moderate computational resources, along with very fast convergence were indeed observed through the code tests of Section 4. As a matter of fact, the highest truncation orders we have tested $\left(N_{r}=7, N_{x}=5\right)$ resulted in a dynamical system of 48 equations, which can be considered reasonable in the realm of large numerical simulation using Galerkin method [13]. It is important to mention that the radial and angular truncations associated to each Galerkin expansion of the metric functions $\beta, U$ and $S$ (cf. Eqs. (11), (12) and (13)) can be chosen freely starting from their corresponding minimum values to comply with the requirements of stability of weak gravitational waves. In deriving the dynamical system we have set for the sake of convenience $\left(N_{r}^{\beta}=N_{r}-1, N_{x}^{\beta}=N_{x}-1\right)$, $\left(N_{r}^{U}=N_{r}+2, N_{x}^{\beta}=N_{x}+1\right)$ and $\left(N_{r}^{S}=N_{r}+1\right.$, $\left.N_{x}^{S}=N_{x}+2\right)$. 


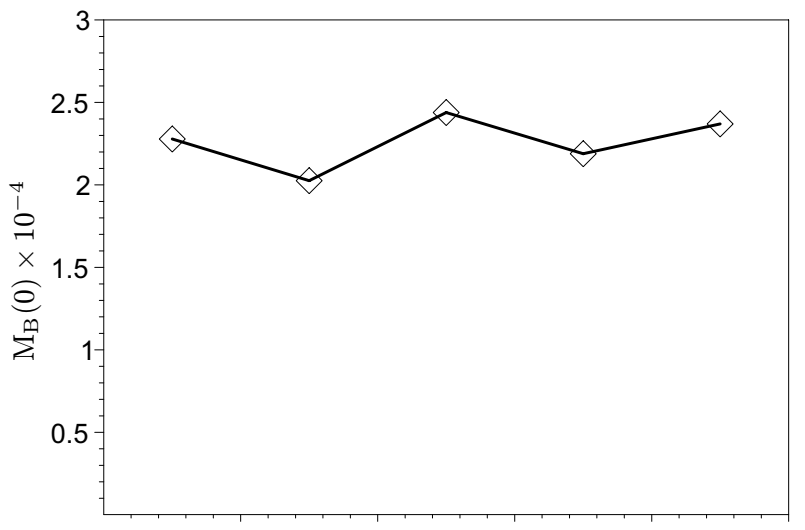

(a)

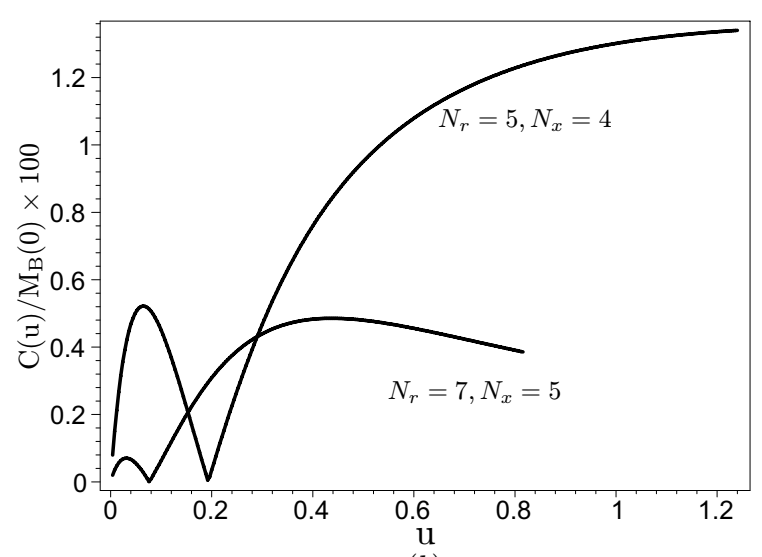

(b)

Figure 3 - (a) Sequence of the initial values of the Bondi mass (cf. Eq. (26)) corresponding to the initial data (27) with $A_{0}=0.025$ and for $\left(N_{r}=3, N_{x}=\right.$ 3), $\left(N_{r}=4, N_{x}=4\right),\left(N_{r}=5, N_{x}=4\right),\left(N_{r}=6, N_{x}=4\right)$ and $\left(N_{r}=7, N_{x}=5\right)$ read from the left to the right, respectively. The convergence towards the actual value for the initial mass is indicated. (b) The relative error $|C(u)| / M_{B}(0) \times 100$ in the global energy conservation is evaluated up to $u=1.2$, where the dynamical system was integrated with stepsize of $10^{-3}$. Note that for $N_{r}=7, N_{x}=5$ the energy conservation is attained to about $0.45 \%$ accuracy.

Possibly the evaluation of a large number of spatial integrations as demanded by the projections of the field equations onto the angular and radial basis functions might be seen as the major computational drawback of the present scheme. In particular, the increase of the truncation orders produces a dramatic growth of the number of such spatial integrations due to the nonlinear terms present in the field equations. In order to circumvent this potential problem we have developed efficient symbolic routines in Maple for these operations, which proved to be low-cost computationally. We have also constructed a 4th-order Runge-Kutta integrator to evolve the dynamical system (19), which is adapted to solve numerically the constraint equations derived from the hypersurface equations at each time step of integration.

There are some important further directions of this work that are currently in progress. We mention the extension of the present code to encompass the full nonlinearities of the field equations. Also, we may consider the field equations in the gauge $K=L=0$ that can be done after a change in the coordinates $u, \theta$ followed by a rescale of the radial coordinate [3]. In this case distinct radial basis functions for $\gamma, U$ and $V$ have been already determined, and this particular gauge will provide a simpler the evaluation of the mass function and the news function.

\section{APPENDICE A: RADIAL BASIS FUNCTIONS}

We shall present the radial basis functions $\Psi_{k}^{(\gamma)}, \Psi_{k}^{(U)}$ and $\Psi_{k}^{(\beta)}$ which are constructed using linear combinations of the rational Chebyshev polynomials [11] $T L_{k}(r)$ defined in the semiinfinite range $0 \leq r<\infty$. First it is necessary to define an auxiliary function $\psi_{k}(r)$

$$
\psi_{k}(r)=T L_{k+1}(r)+T L_{k}(r) .
$$

The radial basis functions for the decompositions of $\gamma, U$ or $S$ and $\beta$ are given by

$$
\begin{gathered}
\Psi_{k}^{(U)}(r)=\frac{1}{2} \psi_{k}(r) \\
\Psi_{k}^{(\gamma)}(r)=\frac{1}{4}\left(\frac{1+2 k}{3+2 k} \psi_{k+1}(r)+\psi_{k}(r)\right), \quad \text { (A3) } \\
\Psi_{k}^{(\beta)}(r)=\frac{1}{4} \psi_{k}(r) \\
+\frac{\left(2 k^{3}+14 k^{2}+25 k+9\right)}{4\left(7+6 k+k^{2}\right)(k+3)} \psi_{k+1}(r) \\
-\frac{3(k+1)}{4\left(k^{2}+6 k+7\right)(k+3)(2 k+7)} \psi_{k+2}(r) \\
-\frac{(k+1)\left(4 k^{4}+38 k^{3}+118 k^{2}+137 k+48\right)}{4\left(k^{2}+6 k+7\right)(k+3)(k+4)(2 k+7)} \psi_{k+3}(r) \\
-\frac{(k+1)(3+2 k)\left(2+4 k+k^{2}\right)(k+2)}{4\left(7+6 k+k^{2}\right)(k+3)(k+4)(2 k+7)} \psi_{k+4}(r) \quad \text { (A4) }
\end{gathered}
$$

which reproduces correctly the boundary conditions at $r=0$ and $r \rightarrow \infty$.

\section{ACKNOWLEDGMENTS}

The authors acknowledge the financial support of CNPq and CAPES. 


\section{REFERENCES}

[1] EINSTEIN A. 1916. Die Grundlage der allgemeinen Relativitätstheorie (The foundation of General Relativity). Annalen der Physik 49.

[2] BONDI H. 1960. Gravitational waves in general relativity. Nature, 186: 535.

[3] BONDI H, VAN DER BURG MGJ \& METZNER AWK. 1962. Proc. R. Soc. London, A, 269: 21.

[4] WINICOUR J. 2005. Characteristic Evolution and Matching. Living Rev. Relativity, 8: 10.

[5] YORK JW. 1979. Kinematics and dynamics of general relativity, in L.L. Smarr, ed., Sources of gravitational radiation. Proceedings of the Battelle Seattle Workshop, 1978, 83-126. (Cambridge University Press).

[6] ISAACSON RA, WELLING JS \& WINICOUR J. 1983. J. Math. Phys., 24(7): 1824.

[7] GÓMEZ R, PAPADOPOULOS P \& WINICOUR J. 1994. J. Math. Phys., 35(8): 4184.

[8] STEWART JM. 1989. Proc. R. Soc. London, Ser. A, 424: 211.

[9] d'INVERNO RA \& VICKERS JA. 1997. Phys. Rev. D, 54(8): 4919 (1996). Phys. Rev. D, 56(2): 772.

[10] GOTTLIEB D \& ORSZAG SA. 1995. Numerical Analysis of Spectral Methods: Theory and Applications (Society for Industrial and Applied Mathematics, Philadelphia, U.S.A. 1977); C. Canuto, M.Y. Hussaini, A. Quarteroni and T.A. Zang, Spectral Methods in Fluid Dynamics (SpringerVerlag, Berlin, Germany; Heidelberg, Germant, 1988); Spectral Methods:
Fundamentals in Single Domains (Springer-Verlag, Berlin, Germany; Heidelberg, Germant, 2006); Spectral Methods: Evolution to Complex Geometries and Applications to Fluid Dynamics (Springer-Verlag, Berlin, Germany; Heidelberg, Germant, 2007); B. Fronberg, Pratical Guide to Pseudospectral Methods, Cambridge University Press (Cambridge, U.K.).

[11] BOYD J. 2001. Chebyshev and Fourier spectral methods, $2^{\text {nd }}$ ed. (Dover, New York).

[12] BONAZZOLA S, GOURGOULHON E \& MARK JA. 1999. Spectral Methods in General Relativistic Astrophysics. J. Comp. and Applied Math., 109: 433 (1999); P. Grandclément and J. Novak, Spectral Methods for Numerical Relativity, arXiv:0706.2286v1 [gr-qc], and all references therein.

[13] HOLMES P, LUMLEY JL \& BERKOOZ G. 1998. Turbulence, Coherent Structures, Dynamical Systems and Symmetry, Cambridge University Press (Cambridge).

[14] SIEBEL F. 2002. Simulation of axisymmetric flows in the characteristic formulation of general relativity. Ph.D. Thesis, (Technische Universität München, München, Germany).

[15] GÓMEZ R, BARRETO W \& FRITTELLI S. 2007. A framework for large-scale relativistic simulations in the characteristic approach, gr-qc 0711.0564 .

[16] GÓMEZ R, REILLY P \& WINICOUR J. 1993. Phys. Rev. D, 47: 3292.

[17] OLIVEIRA HP, RODRIGUES EL, SOARES ID \& TONINI EV. 2007. Int. J. Mod. Phys. C, 18(12): 1839 (preprint gr-qc/0703007). 